\title{
New class of compact diode pumped sub 10 fs lasers for biomedical applications
}

Le, T.; Mueller, A.; Sumpf, B.; Jensen, Ole Bjarlin; Hansen, Anders Kragh; Andersen, Peter E.

Published in:

Frontiers in Ultrafast Optics: Biomedical, Scientific, and Industrial Applications XVI

Link to article, DOI:

$10.1117 / 12.2208589$

Publication date:

2016

Document Version

Publisher's PDF, also known as Version of record

Link back to DTU Orbit

Citation (APA):

Le, T., Mueller, A., Sumpf, B., Jensen, O. B., Hansen, A. K., \& Andersen, P. E. (2016). New class of compact diode pumped sub 10 fs lasers for biomedical applications. In Frontiers in Ultrafast Optics: Biomedical, Scientific, and Industrial Applications XVI (Vol. 9740). [97400D] SPIE - International Society for Optical Engineering. Proceedings of SPIE - The International Society for Optical Engineering https://doi.org/10.1117/12.2208589

\section{General rights}

Copyright and moral rights for the publications made accessible in the public portal are retained by the authors and/or other copyright owners and it is a condition of accessing publications that users recognise and abide by the legal requirements associated with these rights.

- Users may download and print one copy of any publication from the public portal for the purpose of private study or research.

- You may not further distribute the material or use it for any profit-making activity or commercial gain

- You may freely distribute the URL identifying the publication in the public portal 


\title{
New class of compact diode pumped sub 10 fs lasers for biomedical applications
}

\author{
T. Le ${ }^{1 * a}$, A. Müller ${ }^{b}$, B. Sumpf ${ }^{b}$, O. B. Jensen ${ }^{\mathrm{c}}$, A. K. Hansen ${ }^{\mathrm{c}}$ P. E. Andersen ${ }^{\mathrm{c}}$, \\ ${ }^{a}$ Femtolasers, Fernkorngasse 10, 1100 Vienna, Austria; ${ }^{b}$ Ferdinand-Braun-Institut für \\ Höchstfrequenztechnik, Gustav-Kirchoff-Strasse 4, 12489 Berlin, Germany; ${ }^{\circ}$ DTU Fotonik, \\ Department of Photonics Engineering, Technical University of Denmark, Frederiksborgvej 399, \\ 4000 Roskilde, Denmark
}

\begin{abstract}
Diode-pumping Ti:sapphire lasers promises a new approach to low-cost femtosecond light sources. Thus in recent years much effort has been taken just to overcome the quite low power and low beam qualities of available green diodes to obtain output powers of several hundred milliwatts from a fs-laser. In this work we present an alternative method by deploying frequency-doubled IR diodes with good beam qualities to pump fs-lasers. The revolutionary approach allows choosing any pump wavelengths in the green region and avoids complicated relay optics for the diodes. For the first time we show results of a diode-pumped 10 fs-laser and how a single diode setup can be integrated into a $30 \times 30 \mathrm{~cm}^{2} \mathrm{fs}-l$ aser system generating sub 20 fs laser pulses with output power towards half a Watt. This technology paves the way for a new class of very compact and cost-efficient fs-lasers for life science and industrial applications.
\end{abstract}

Keywords: Tapered diode lasers, Second harmonic generation, Ti:sapphire lasers, ultrafast lasers, femtosecond lasers

\section{INTRODUCTION}

The fs-laser has become an irreplaceable light source for a vast number of applications in research, medicine, and security. In particular mode-locked Ti:sapphire lasers have found widest penetration into the commercial fs-laser market. Due to better thermal and material properties of sapphire over many other host media, Ti:sapphire lasers are regarded as the ultimate light source in terms of power, pulse duration and stability. They even come as turn-key devices that can work in difficult environments. However, in the majority of cases they are still a big cost factor in their role as a light source. Since first ultrafast lasers commercially became available in the early 1990s the basic principle of how a modelocked laser delivers femtosecond pulses has not undergone ground-breaking changes in the sense, that their size, performance and cost still depend on power, size and cost of the available pump lasers. Starting with large frame Ar-Ion lasers at the beginning pump lasers nowadays based on diode-pumped frequency doubled Nd:Vanadate or Nd:YAG laser systems that are entirely solid-state and much more compact. But in fact, multiple wavelength conversion units, moderate electro-optical efficiencies and some level of complexity result in demanding manufacturing efforts, heavy and large device dimensions and water cooling requirements. Thus size and cost of a femtosecond Ti:sapphire laser primarily depend on the availability and technology of the pump laser.

Since the challenge to generate powerful continuous wave green light with diffraction-limited beam quality at small cost and dimension has not been accepted for a long time alternative light sources to the Ti:sapphire laser came up to existence. For instance femtosecond fiber lasers pumped with laser diodes deliver several $100 \mathrm{~mW}$ using an amplifying stage. Although power and pulse duration are limited by fiber non-linearities they have started to replace Ti:sapphire lasers in many areas like $\mathrm{THz}$ generation or two-photon polymerization. However, Erbium doped fibers have to be frequency doubled thus limiting their usefulness in the $800 \mathrm{~nm}$ wavelength range where biological tissues have less absorption or common photo-conductive switches for $\mathrm{THz}$ generation are readily available. Also in optical coherence tomography the Ti:sapphire laser had been replaced by very cost-effective super-luminescence light emitting diodes which fortunately accelerated the market penetration of OCT devices rapidly ${ }^{1}$. Also wavelength swept sources based on semiconductor optical amplifiers at $1050 \mathrm{~nm}$ enabling better penetration and faster scanning are gaining more relevance ${ }^{2}$.

\footnotetext{
${ }^{1}$ tuan.le@femtolasers.com; phone: +431503700248; www.spectra-physics.com
} 
But none of these light sources can fully replace the femtosecond Ti:sapphire laser providing the highest axial resolution in OCT or the broadest $\mathrm{THz}$ spectra ${ }^{3,4}$.

In this work we present latest results of using frequency doubled tapered diode lasers to pump femtosecond Ti:sapphire lasers. The pump laser consists of a spectrally stabilized, high-efficient, high brightness tapered diode laser combined with a periodically poled nonlinear crystal. In contrast to alternative solid-state laser technologies no additional optical resonator is required to achieve SHG efficiencies up to $19 \%$ with one non-linear crystal only in single-pass configuration $^{5}$. As alignment tolerances are significantly relieved compared to a resonator concept, innovative mounting and packaging technologies can be applied, aiming at significantly reduced costs, improved compactness and improved performances with respect to low amplitude noise and long term power stability. Using this concept $82 \mathrm{~mW}$ from a mode-locked Ti:sapphire laser pumped by a single-pass frequency doubled DBR-tapered diode laser was already demonstrated ${ }^{6}$. However, very recently a new concept for power scaling the second harmonic generation by cascading conversion stages was introduced ${ }^{7}$. Using two nonlinear crystals $39 \%$ optical efficiency was achieved from a 9.5 W DBR-tapered diode laser. In this work we report on the first results obtained using a two stage conversion scheme to pump an ultra-broadband Ti:sapphire laser.

\section{EXPERIMENT}

The pump laser consists of a $6 \mathrm{~mm}$ DBR-tapered diode laser generating $10.5 \mathrm{~W}$ of light at $1064 \mathrm{~nm}$. Two separate electrical contacts control the currents through a $4 \mathrm{~mm}$ tapered diode section and half of a $2 \mathrm{~mm}$ ridge wave guide section. The DBR diode laser is mounted p-side up on a CuW heat spreader which is again mounted on a $25 \times 25 \mathrm{~mm}$ conduction cooled package mount. The astigmatic emission from the diode is collimated by an aspheric lens in the fast axis and a cylindrical lens is used to correct the astigmatism and collimate the beam in the slow axis. The collimated beam is then sent through a half-wave plate and an optical isolator to prevent light reflected back to the DBR diode laser. A second half-wave plate is used after the isolator to adjust and optimize the second harmonic generation. For singlepass configuration a lens is used to focus the beam into a $30 \mathrm{~mm}$ PPMgLN crystal with a beam waist diameter of approximately $60 \mu \mathrm{m}$. The crystal is angled cut at $10^{\circ}$, antireflection coated at $1064 \mathrm{~nm}$ and $532 \mathrm{~nm}$ and temperature stabilized at $35^{\circ} \mathrm{C}$. The SHG output power reaches up to $1.7 \mathrm{~W}$. The sealed housing of the DBR diode laser plus conversion stage (laser head) has a foot print of about $140 \mathrm{~mm} \times 89 \mathrm{~mm}$. Emission of this setup is used to pump a standard dispersive mirror based cavity that supports pulse durations down to $10 \mathrm{fs}^{8}$. Our gain medium is a Ti:sapphire crystal with $4 \mathrm{~mm}$ optical path. The absorption is $\alpha=4.25 \mathrm{~cm}^{-1}( \pm 5 \%)$ at $514 \mathrm{~nm}$ with a FOM of $\sim 150$. The pump beam having a collimated diameter of about $2.5 \mathrm{~mm}$ is focused into the crystal with an AR-coated $f=35 \mathrm{~mm}$ lens. Two dichroic curved mirrors are focusing and directing the beam to both arms of the x-folded cavity. The angles are optimized to compensate for astigmatism inside the crystal. All plane mirrors in the fs-laser are negative dispersive supporting reflectivities higher than $99.5 \%$ with an average GVD of about $-40 \mathrm{fs}^{2}$ between $700 \mathrm{~nm}$ to $900 \mathrm{~nm}$. Soft aperture mode-locking is initiated by disturbing one end mirror. The pulse repetition rate that basically determines the pulse energy is set by the length of the cavity. In this work we report on results with a $300 \mathrm{MHz}$ cavity (length $\sim 0.5 \mathrm{~m}$ ) pumped with single-pass SHG and a $125 \mathrm{MHz}$ cavity (length $\sim 1.2 \mathrm{~m}$ ) pumped with double cascaded SHG configuration.

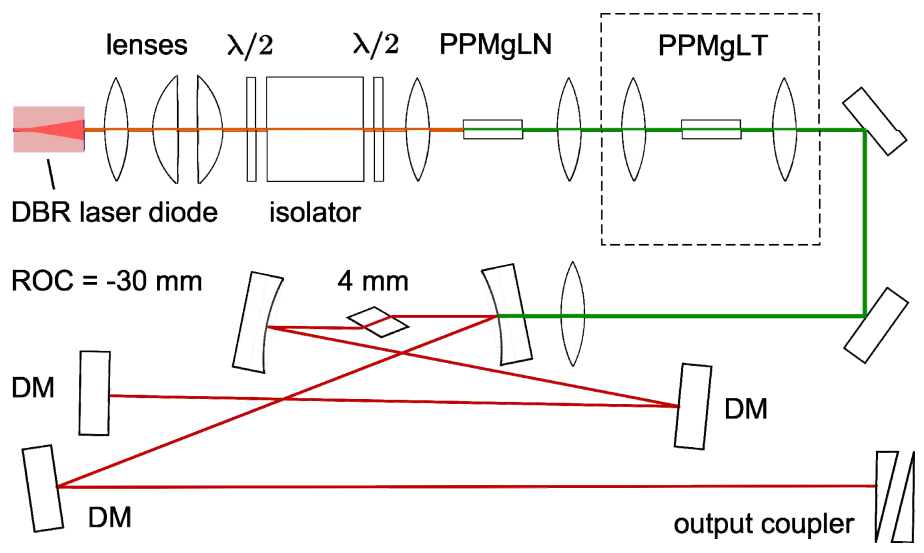

Figure 1: Optical scheme of how fs-laser light is generated starting from a DBR tapered diode laser. The dashed square denotes the additional conversion stage to enhance the second harmonic output. DMs are dispersive mirrors. 
The beam profile of the single-pass configuration is measured using a WinCamD-UCD3 camera from DataRay Inc. Throughout $1 \mathrm{~W}$ to $1.6 \mathrm{~W}$ behind the first SHG stage the beam cross sections show ripples and distinct deviations from axial symmetry (see Figure 2). For comparison a conventional diode pumped solid-state (DPSS) laser (gem 532 from Laser Quantum) is used. Swapping between both pump light sources shows that the pump efficiency is $10 \%$ to $15 \%$ less for the frequency doubled DBR diode laser. However, the structured beam profile is not translated into the femtosecond laser which delivers a smooth intensity profile as normal (Figure 2: right). The beam is used to pump a $300 \mathrm{MHz}$ femtosecond Ti:sapphire laser delivering sub $20 \mathrm{fs}$ pulses. At $1.5 \mathrm{~W}$ more than $190 \mathrm{~mW}$ output power with pulse energies in the order of $\sim 0.65 \mathrm{~nJ}$ is obtained. Figure 3 shows the spectral shape and mode-locked output power of the fslaser recorded over 17 hours. The spectrum corresponds to a pulse duration of about $15 \mathrm{fs}$. Although the FWHM bandwidth is $67 \mathrm{~nm}$ it is perceivable that the spectrum extends from below $700 \mathrm{~nm}$ to more than $900 \mathrm{~nm}$ indicating that
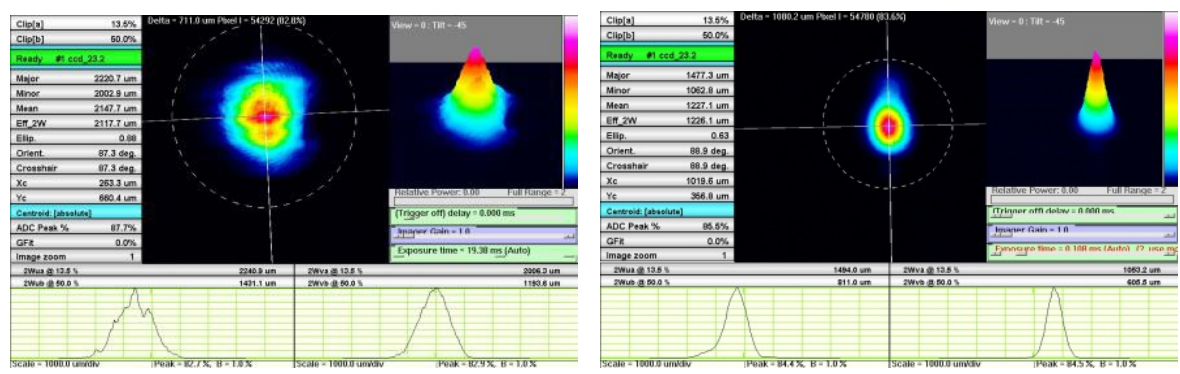

Figure 2: left: Beam profile of the single-pass SHG output measured at different powers; right: beam profile of the modelocked Ti:sapphire laser pumped by the DBR diode laser with a single-pass SHG stage.
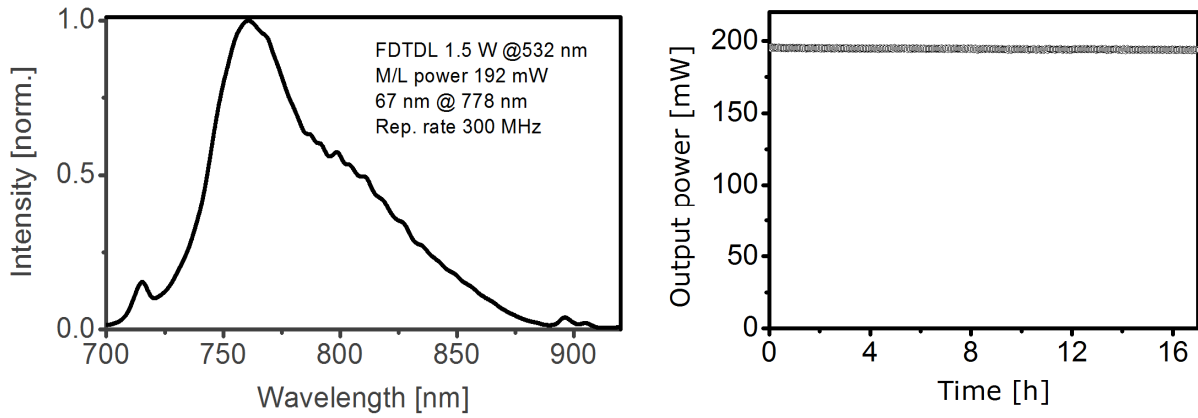

Figure 3: left: spectral intensity of the DBR diode pumped femtosecond laser; right: power monitoring over 17 hours

larger FWHM bandwidths can be accepted. Hence, more effort towards fine-tuning intra-cavity dispersion leads to the formation of a $125 \mathrm{~nm}$ FWHM spectral bandwidth from the Ti:sapphire laser. Although the overall spectral coverage is not extended which is probably restricted by the used dispersive mirrors the shape of the spectrum supports pulse durations below $10 \mathrm{fs}$ as is shown in Figure 4. The average output power of the sub $10 \mathrm{fs}$ laser amounts to about $115 \mathrm{~mW}$.
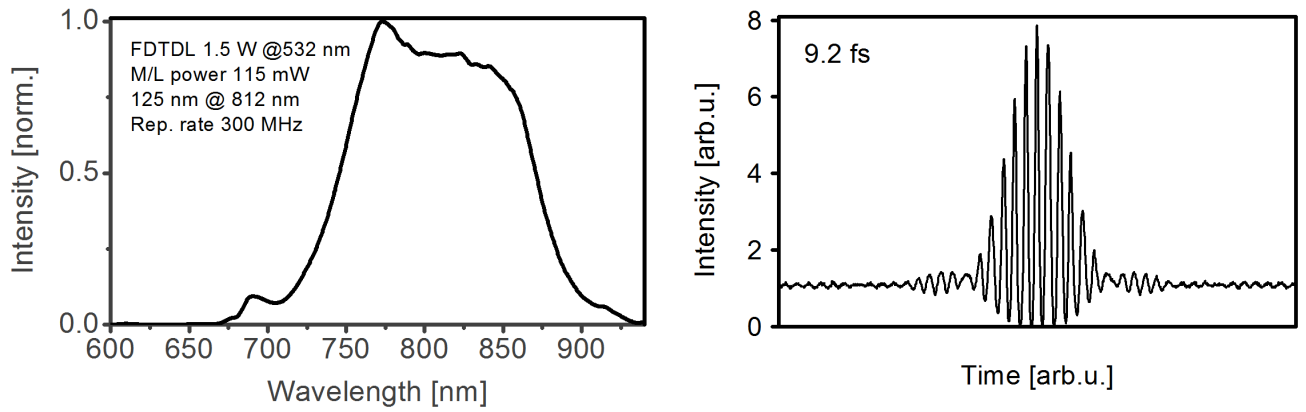

Figure 4: frequency-doubled tapered diode laser (FDTDL) pumps a sub-10 fs Ti:sapphire laser emitting spectral bandwidth of $125 \mathrm{~nm}$; right: measured autocorrelation corresponds to $9.2 \mathrm{fs}$ assuming a hyperbolic secant squared form of laser pulses 
In order to scale up the output power of the fs- laser two SHG stages after the DBR tapered diode laser are applied. The optical setup is similar to what has been reported by Hansen et $\mathrm{al}^{7}$. Using two different crystals whereas the first one is chosen for most efficient second harmonic generation while the second crystal does better handle thermal effects, $2.4 \mathrm{~W}$ of light at $532 \mathrm{~nm}$ is obtained. In order to achieve even higher pulse energies and pulse peak intensities we change the repetition rate of the Ti:sapphire laser to $125 \mathrm{MHz}$ by enlarging its cavity length 2.4 fold. When pumped with $2.4 \mathrm{~W}$ it delivers output powers of $400 \mathrm{~mW}$ in $\mathrm{cw}$ and $320 \mathrm{~mW}$ in mode-locked operation. This is a 1.7 times higher average power output over the single SHG stage case. However, with regard to pulse energy a four-fold improvement is obtained. Figure 5 shows the femtosecond laser and the pump laser on an optical breadboard. The size of the pump laser that includes both single-pass SHG stages is $183 \times 114 \times 50 \mathrm{~mm}^{3}$. Its sealed encasement sits on a water cooled base plate which is temperature stabilized at $20^{\circ} \mathrm{C}$. The same cooling is applied to the femtosecond laser. No additional beam shaping, e.g. with cylindrical lenses, is used. Light pulses from the Ti:sapphire laser have spectral FWHM bandwidth of $36 \mathrm{~nm}$ centered at $816 \mathrm{~nm}$ which corresponds to a pulse duration of about $25 \mathrm{fs}$.
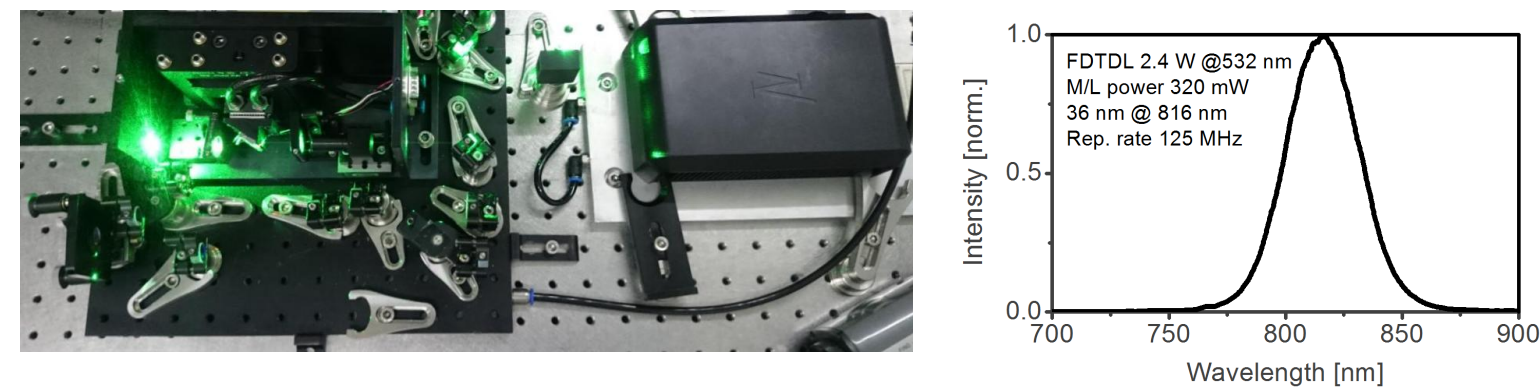

Figure 5: left: laboratory setup of a $125 \mathrm{MHz}$ laser which is pumped by a frequency-doubled tapered diode laser (FDTDL); right: spectral intensity of pulses generated from the FDTDL at $2.4 \mathrm{~W}$

\section{DISCUSSION}

Despite its non-smooth beam profile the frequency doubled diode laser is shown to fully replace solid-state lasers for high demanding applications like pumping femtosecond lasers in the sub 20 fs range. We achieve close to $200 \mathrm{~mW}$ output power with only $1.5 \mathrm{~W}$ from a one SHG stage single-pass configuration. The spectral bandwidth of the femtosecond laser easily supports sub 20 fs laser pulses while its base covers almost the full spectral bandwidth of the used dispersive mirrors. We also saw no significant deviation from a pump beam alignment optimized for a state-of-theart solid-state pump laser, i.e. only very little re-adjustment of the pump lens was required to compensate for different beam divergences of both pump lasers. No compromise in spectral bandwidth and pulse duration is required since even $10 \mathrm{fs}$ laser pulses can be realized. For the first time a sub $10 \mathrm{fs}$ laser is shown to be pumped directly by a single tapered diode laser. This result paves the way for very cost effective high-end femtosecond lasers for being used in applications that basically need a few $\mathrm{mW}$ impinging on the region of interest like multi-photon microscopy, $\mathrm{THz}$ spectroscopy or optical coherence tomography. Such a fs-laser has already been employed by König et al. ${ }^{9}$ inside a commercial multiphoton tomograph and successfully imaged autofluorescence signals from several unstained biological samples, i.e. human skin, adherent cells and epithelial layers as well as stroma of cornea samples. Hence, tapered diode lasers with simple external frequency conversion stages could simply replace bulky and expensive solid-state lasers to finally make affordable fs-lasers possible. Currently the size of this pump laser is mainly determined by the size of the optical isolator. Further development on better robustness of the diode laser to back reflection could avoid necessity of optical isolators and bring down the size of the laser head packaging considerably since the length of the DBR diode laser is only $6 \mathrm{~mm}$. For the first time a cascaded frequency conversion scheme is used to pump a femtosecond Ti:sapphire laser. Although $320 \mathrm{~mW}$ marks the highest mode-locked output power ever recorded from a fs-laser pumped by a tapered diode laser we assume that the efficiency can be improved by further optimizing the beam quality emerging from the SHG stages. The moderate efficiency might be caused by thermal effects or formation of astigmatism which has to be reinvestigated. However, due to the larger output power and lower repetition rate the Ti:sapphire laser delivers pulses already reaching $100 \mathrm{~kW}$ peak intensities. The laser head can be designed to have a $285 \times 285 \mathrm{~mm}^{2}$ foot print which already includes the "high power" tapered diode laser. Figure 6 shows how the head size of the femtosecond laser changes over the standard version with a single SHG stage after the tapered diode laser. 

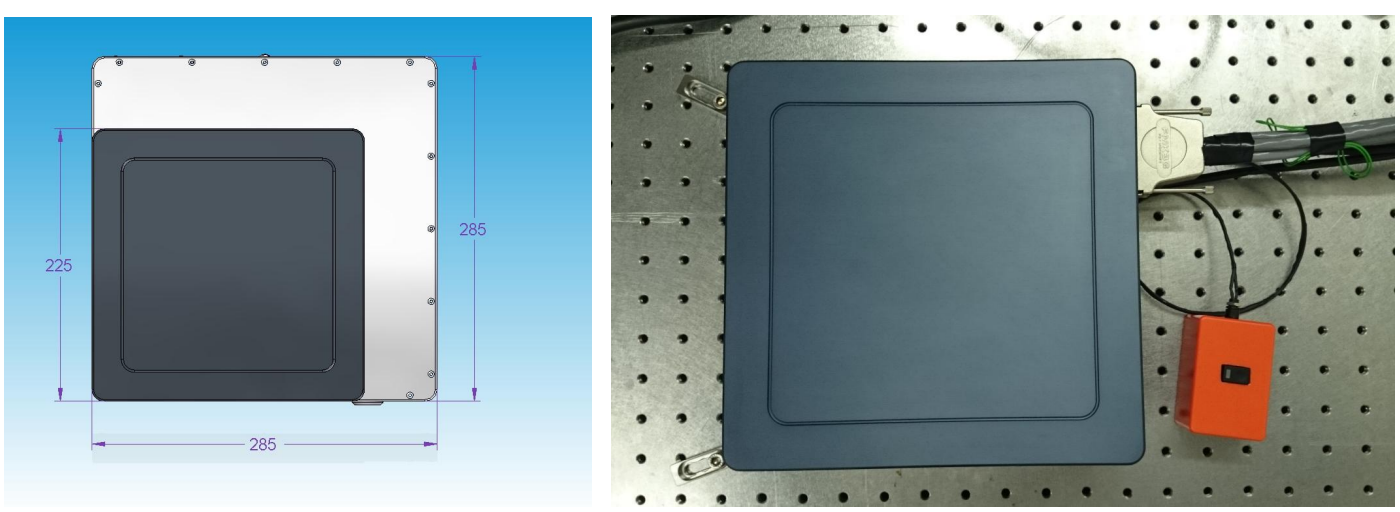

Figure 6: left: size of the laser head containing the $125 \mathrm{MHz}$ Ti:sapphire laser and the "high-power" frequency doubled diode laser (white) compared to the very compact $300 \mathrm{MHz}$ version with a standard $1.5 \mathrm{~W}$ version of the pump laser; right: a compact $300 \mathrm{MHz}$ version on an optical breadboard. The orange box simply holds a button to initiate mode-locking of the femtosecond laser.

\section{SUMMARY}

Frequency doubled diode lasers are very promising candidates to replace existing bulky and expensive lasers used to pump femtosecond lasers. Particularly Ti:sapphire lasers provide very short light pulses and will immensely benefit from new pump light sources since they deliver high light peak intensities even at lowest average powers. This new class of pump laser even meets the criteria to produce fs-lasers providing sub $10 \mathrm{fs}$ pulses. We have shown that a laser head of the size of a sheet of paper is able to generate $>190 \mathrm{~mW}$ of optical pulses in the sub 20 fs range. By a small enlargement of the foot print, i.e. $25 \%$ on both sides, the power is increased to $320 \mathrm{~mW}$ with even 4 times higher pulse energies. However, we guess that this is only the first stage of what can be expected from performances of sub- 20 fs or sub- 10 fs lasers pumped with this novel kind of lasers. Hansen et al. ${ }^{7}$ already reported about $3.7 \mathrm{~W}$ diffraction limited green light $\left(M^{2}=1.25\right)$ from a two stage cascading configuration while Müller et al. ${ }^{10}$ could demonstrate up to $4 \mathrm{~W}$ green light by sum-frequency generation of spectrally combined tapered diode lasers. Combining both schemes Hansen et al. ${ }^{11}$ recently showed that $5.5 \mathrm{~W}$ can be reached with conversion efficiencies up to $50 \%$. Hence, the technology to frequency convert high-end or so-called "high-brightness" laser diodes has opened up a new route to very compact and powerful pump lasers that facilitate very compact and cost-effective sub $20 \mathrm{fs}$ lasers in the $0.5 \mathrm{~W}$ or even several Watts range.

Acknowledgement: This work has received funding from the European Union's Seventh Framework Programme (FAMOS, FP7-ICT-2011-3-5a) under grant agreement $\mathrm{n}^{\circ}$ FP7-ICT-317744. 


\section{REFERENCES}

[1] Stephen G. Anderson, "Optical Coherence Tomography,", SPIE professional, January 2014, $<$ https://spie.org/membership/spie-professional-magazine/spie-professional-archives-and-special-content/spieprofessional-archives/archived-issues/2014_jan_archive_spie_pro/2014-january/featuredarticles/oct_anderson?ArticleID=x105085,">

[2] Krstajić, N.; Childs, D.T.D.; Matcher, S.J.; Livshits, D.; Shkolnik, A.; Krestnikov, Igor; Hogg, R.A., "SweptSource Laser Based on Quantum-Dot Semiconductor Optical Amplifier-Applications in Optical Coherence Tomography," in Photonics Technology Letters, IEEE, vol.23, no.11, pp.739-741 (2011)

[3] Kampfrath, Tobias and Nötzold, Jan and Wolf, Martin, "Sampling of broadband terahertz pulses with thick electro-optic crystals," Applied Physics Letters, 90, 231113 (2007)

[4] Prashanth C. Upadhya, Wenhui Fan, Andrew Burnett, John Cunningham, A. Giles Davies, Edmund H. Linfield, James Lloyd-Hughes, Enrique Castro-Camus, Michael B. Johnston, and Harvey Beere, "Excitation-densitydependent generation of broadband terahertz radiation in an asymmetrically excited photoconductive antenna," Opt. Lett. 32, 2297-2299 (2007)

[5] Ole Bjarlin Jensen, Peter E. Andersen, Bernd Sumpf, Karl-Heinz Hasler, Götz Erbert, and Paul Michael Petersen, "1.5 W green light generation by single-pass second harmonic generation of a single-frequency tapered diode laser," Opt. Express 17, 6532-6539 (2009)

[6] André Müller, Ole Bjarlin Jensen, Angelika Unterhuber, Tuan Le, Andreas Stingl, Karl-Heinz Hasler, Bernd Sumpf, Götz Erbert, Peter E. Andersen, and Paul Michael Petersen, "Frequency-doubled DBR-tapered diode laser for direct pumping of Ti:sapphire lasers generating sub-20 fs pulses," Opt. Express 19, 12156-12163 (2011)

[7] A. K. Hansen, M. Tawfieq, O. B. Jensen, P. E. Andersen, B. Sumpf, G. Erbert, and P. M. Petersen, "Concept for power scaling second harmonic generation using a cascade of nonlinear crystals," Opt. Express 23, 1592115934 (2015)

[8] A Xu, L., A Tempea, G., A Poppe, A., A Lenzner, M., A Spielmann, C., A Krausz, F., A Stingl, A., A Ferencz, K., "High-power sub-10-fs Ti:sapphire oscillators," Applied Physics B Lasers and Optics 65, Number 2, Page 151 (1997)

[9] König, K., Andersen, P., Le, T. and Breunig, H. G., "Multiphoton imaging with a novel compact diode-pumped Ti:sapphire oscillator," Microsc. Res. Tech. 78, 1154-1158 (2015)

[10] André Müller, Ole Bjarlin Jensen, Karl-Heinz Hasler, Bernd Sumpf, Götz Erbert, Peter E. Andersen, and Paul Michael Petersen, "Efficient concept for generation of diffraction-limited green light by sum-frequency generation of spectrally combined tapered diode lasers," Opt. Lett. 37, 3753-3755 (2012)

[11] Anders K. Hansen, Peter E. Andersen, Ole B. Jensen, Bernd Sumpf, Götz Erbert, and Paul M. Petersen, "Highly efficient single-pass sum frequency generation by cascaded nonlinear crystals," Opt. Lett. 40, 5526-5529 (2015) 